\title{
Formation of Stripes and Channels Structure of DPPC Langmuir Blodgett Monolayer at Middle Surface-Pressure
}

\author{
Zhonghui Xue ${ }^{1^{*}}$, Ning Xue ${ }^{2}$ \\ ${ }^{1}$ Shanghai Publishing and Printing College, Shanghai, China \\ ${ }^{2}$ Northeastern University, Qinhuangdao, China \\ Email: *hnlgxzh@163.com
}

How to cite this paper: Xue, Z.H. and Xue, N. (2019) Formation of Stripes and Channels Structure of DPPC Langmuir Blodgett Monolayer at Middle Surface-Pressure. Open Access Library Journal, 6: e5552.

https://doi.org/10.4236/oalib.1105552

Received: June 18, 2019

Accepted: August 4, 2019

Published: August 7, 2019

Copyright $\odot 2019$ by author(s) and Open Access Library Inc.

This work is licensed under the Creative Commons Attribution International License (CC BY 4.0).

http://creativecommons.org/licenses/by/4.0/

(c) (i) Open Access

\begin{abstract}
Monolayers of L- $\alpha$-Dipalmitoylphosphatidylcholine (DPPC) on the air-water interface have been transferred at a middling applied surface-pressure onto the mica substrates using the Langmuir-Blodgett (LB) technique. AFM was applied to observe the formation of morphology of DPPC. Our results show that the Langmuir-Blodgett monolayers of DPPC on mica substrates generate a structured surface with periodic channels and stripes. The LB monolayers obtained at a surface pressure correspond to $20 \mathrm{mN} / \mathrm{n}$. We take into account several explanations to understand the observed surface topological views. On the other hand, a lot of lipids, their Langmuir monolayer and Langmuir-Blodgett monolayer are excellent models of half-layer biological membranes, which can provide closer biological environment. Therefore, using the lipid Langmuir-Blodgett or Langmuir monolayer as prototype, one can simulate further biological environment and make further investigation on biomineralization mechanism of template-controlled.
\end{abstract}

Subject Areas
Analytical Chemistry

Keywords

Langmuir Monolayers, Stripes, Channels, DPPC

\section{Introduction}

Phospholipid bilayers are the matrix of biological membranes. It is well known that phospholipid, owing to amphiphilic character, is able to form stable mono- 
layers at the air-water interface [1]. Therefore, phospholipid monolayers can be considered as a simple model system for biological membranes to study the biomembrane mimetic systems [2]. For example, phospholipid Langmuir monolayers or Langmuir-Blodgett monolayers can be used as a template to control the growth of inorganic materials [3]. A structure characterization of the LB monolayers is essential in order to use them in model studies or as functional templates. From these reasons, phospholipid monolayers, both Langmuir monolayers and Langmuir-Blodgett monolayers, have been widely characterized using a variety of techniques [4]-[10]. Among of them, AFM has been shown as a powerful tool in providing molecular scale topographic views of a variety of materials ranging from solid surfaces to organic thin films and biological systems [11] [12].

Nano-lath lattices consisting of stripes and channels can be produced by using physical or chemical methods, such as microcontact printing [13] [14] [15], micromachining [16], photolithography [17] [18], and vapor depositon [19]. Another strategy for introducing anisotropic texture is based on the use of the intrinsic material properties of stretched ultrathin polymer coatings [20]. At 1992 years Riegler H. et al., 1996 years Yuan Chunbo et al. and 2000 years M. Gleche et al. respectively produced stripes domain structure and stripes and channels with periodic structure of L- $\alpha$-Dipalmitoylphosphatidylcholine Langmuir-Blodgett monolayer film [21] [22] [23]. Riegler H. et al. added the dye molecules into the DPPC chloroform solution observed by fluorescence microscopy, finding, Yuan Chunbo et al. made use of Lanthanide ion to induce formation of stripes domain structure, and M. Gleche et al. applied rapidly $(60 \mathrm{~mm} / \mathrm{min})$ withdrawing a mica by LB technique at very low surface pressure (about 3 $\mathrm{mN} / \mathrm{m}$ ) without adding any ion to the subphase solution. Here, we study the topology of DPPC Langmuir-Blodgett monolayers at surface-pressures equal to 20 $\mathrm{mN} / \mathrm{m}$ with the pure water subphase, the results show that the DPPC could form a surface structure consisting of stripes and channels with a slowly $(5 \mathrm{~mm} / \mathrm{min})$ withdrawing a mica substrate from the interface of monolayers-water at middle surface pressure. In this paper, the surface structure of the DPPC LB monolayers is studied by atomic force microscopy (AFM) at dynamic force mode.

\section{Experiment}

L- $\alpha$-Dipalmitoylphosphatidylcholine (DPPC, Sigma Co.) monolayer was spread from chloroform solution ( $\left.1 \mathrm{mM} \cdot \mathrm{L}^{-1}\right)$ into aqueous triply distilled de-ionized water in a KSV 5000 Langmuir-Blodgett trough (KSV Instruments, Finland). The $\mathrm{pH}$ of the subphase solution is about $5.8-6.0$. The temperature was maintained at $25^{\circ} \mathrm{C}$ with an accuracy of $\pm 0.5^{\circ} \mathrm{C}$. The surface pressure (Ps) was measured with a precision of $0.1 \mathrm{mN} \cdot \mathrm{m}^{-1}$. The DPPC monolayer was compressed at a controlled rate to a pre-specific surface pressure and transferred to a freshly cleaved, hydrophilic mica substrate by vertical dipping at a speed of $5 \mathrm{~mm} \cdot \mathrm{min}^{-1}$. Transfer ratios were approximately unity monitored by the computer system. 
AFM measurements were carried out by using a commercial system (SPA400, Seiko. Instruments Inc., Japan) in the dynamic force mode in air. The scanner used is $100 \mu \mathrm{m}$. Soft cantilever is long with an integrated pyramidal silicon tip, exhibiting a spring constant of $17.5 \mathrm{~N} / \mathrm{m}$.

\section{Results and Discussions}

\subsection{Formation of Films in Aqueous Solution}

Figure 1 displays the pressure-area isotherm curves of the DPPC monolayer spreading on a subphase of distilled water. The pressure-area isotherm of DPPC is coincident with previously published results [24] [25]. Obviously, with the increasing the pressure, the compressibility of LB monolayers decrease gradually. When the surface-pressure is equal to $20 \mathrm{mN} / \mathrm{m}$, the DPPC Langmuir-Blodgett monolayers stand in a liqiud-condensed (LC) phase.

In this case, the Langmuir monolayers have a little compresibility, therefore, the monolayers have a stable state, which provides a good condition to withdraw the DPPC Langmuir monolayers to form a compact LB monolayers from the interface of monolayers/water.

\subsection{AFM Characterization}

Figure 2 shows a series of AFM images of a DPPC monolayer transferred onto mica substrate, which is obtained at two different scales respectively. We have observed a superstructure consisting of stripes and channels with periodicity at the larger scale as shown in Figure 2(a). These images clearly show two distinct height regions. The higher regions are brighter and can be designated the solid

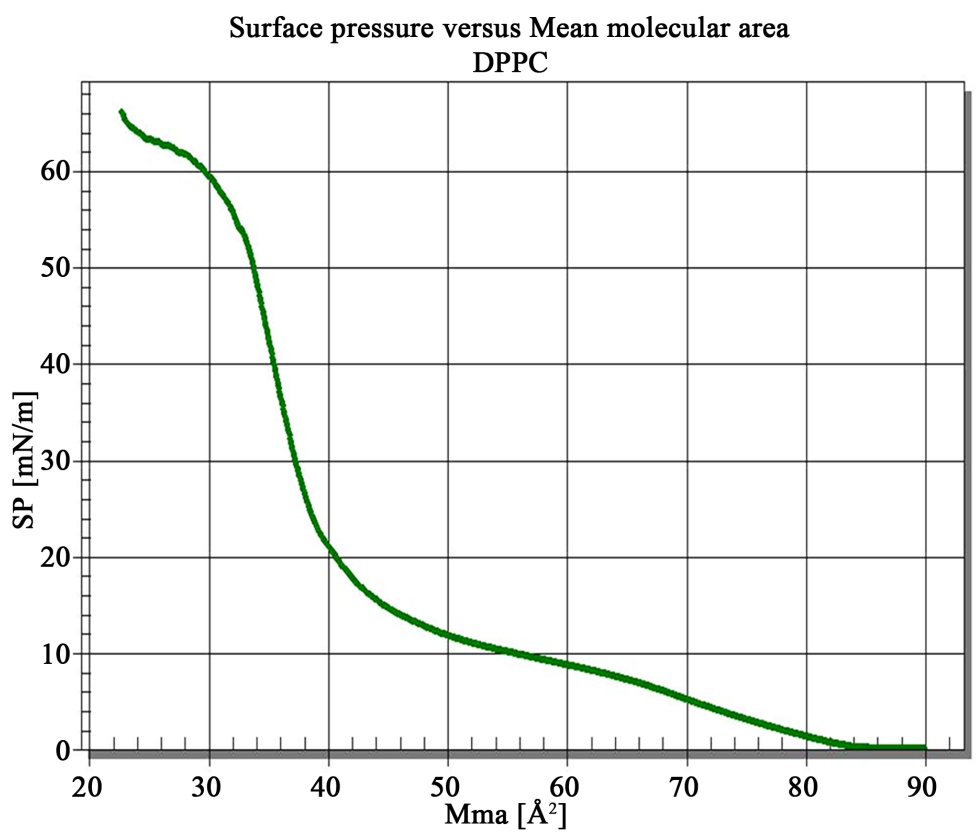

Figure 1. Surface pressure-area ( $\Pi$-A) isotherms of the DPPC monolayers on triply distilled water. 


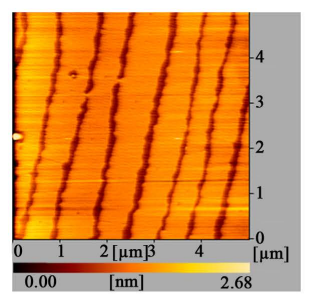

(a)

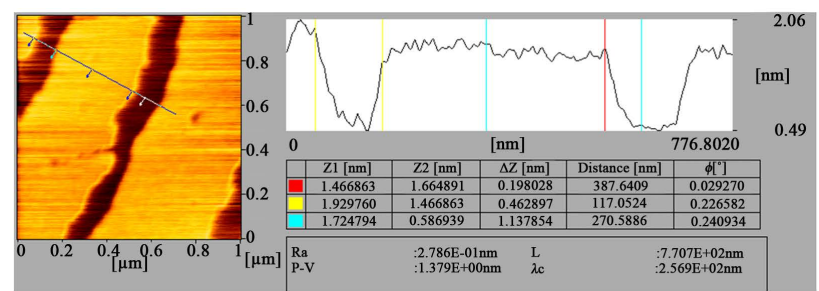

(b)

Figure 2. Series of AFM images of DPPC monolayer transferred onto mica substrates with different scale pressure. Corresponding to $5 \mu \mathrm{m}$ (a); $1 \mu \mathrm{m}$ (b) including the height profile respectively.

phase, while the dark regions represent the liquid phase. From the alternant regions of light and shade, surface topography inspections with an atomic force microscopy reveal regular channels of about $120 \mathrm{~nm}$ in width, which are separated by regular stripes of about monolecular height and a latitude of about 400 $\mathrm{nm}$ as shown in Figure 2(b). These stripes are more closely packed than that observed at low pressures (data no given). Using AFM we can measure the height difference between the stripes regions and channels region in a monolayer. The measurement results show that their height difference is about $1.2 \mathrm{~nm}$, which is less than the length of DPPC molecule $2.8 \mathrm{~nm}$. Also, we note that the atomic level flat mica substrate used here has no effect on this height measurement. Thus, it can be inferred that the molecules in solid phase are standing straight up, while those in the liqiud phase are not lying close to substrate surface but forming a tilt angle with both alkyl-chains on the water surfaces.

How can we understand the stripes and channels deposition? The answer can be found in how the equilibrium meniscus height is related to the surface pressure, the various interfacial energies and the electrostatic interactions et al. In equilibrium, the meniscus height is given by the competition between minimizing the interfacial energies and the hydrostatic energy. In details, several explanations have to be taken into account to understand the observed periodic two-dimension lattices consisting of stripes and channels. It is well known that monolayers of an amphiphilic organic molecule spread at air-water interface can be tranferred during a constant vertical upstroke of a solid substrate immersed in the subphase (hydrophilic deposition). The transfer behaviour of monolayers onto solid substrates depends strongly on the interaction of amphiphilic molecule with the substrate [26]. The strongly interaction will result in a repid adherence of the molecules, causing a reduction in the surface energy [21] and an increase of the contact angle as described by the well known Young's equation. Thus, these alterations are caused by monolayer/substrate interactions which facilitate the condensation of the monolayer into a higher ordered phase. As a consequence, in a stationary configuration the equilibrium meniscus height is lowered. On the other hand, the meniscus height will tend to increase due to the continuous solid substrates motion (upstroke) resulting in an acceletated molecular adsorption on the sbustrate [27]. Thus, the dynamic behaviour of the 
miniscus height is controlled by two counteracting processes, which may induce the Langmuir-Blodgett to form a nano-lath lattices consisting of stripes and channels when it was transferred onto the hydrophilic mica substrates. Additionally, it is well known that anisotropic domain growth at air-water interface can also lead to stripe patterns at a high-pressure, which can be understood in the terms of a lowered line tension competing with the dipole-dipole interactions [28]. Moreover, long-range electrostatic dipole repulsion between DDPC molecules have led these nano-stripes to repel one another and form a superstructure phase with regular nano-channels [22]. Finally the interfacial binding energies between the monolayers/water is more than that of between the monolayers/mica substrates. Thus, it is proposed that monolayer/substrates interactions can lower the free energy of monolayers compared to the aqueous subphase, and thus facilitate its condensation onto the substrates. By reviewing of the above factors, we consider the surface structure consisting of stripes and channels was controlled by several factors through their interactions each other.

This structured surface can be used as template to control the inorganic growth, such as calcium carbonate, calcium phosphate and the other inorganic materials by electrostatic interactions and capillary et al. Our group will further study the potential use of the nano-lath lattices in our followed work.

\section{Conclusions}

We have used AFM to investigate the surface structure of

L- $\alpha$-Dipalmitoylphosphatidylcholine LB monolayers on mica substrates. The formation of nano-lath lattices consisting of stripes and channels was observed. The height difference between the stripes and channels (about $1.2 \mathrm{~nm}$ ) shows that the DPPC molecules in the solid phase are standing straight up, while those in the liquid phase are not lying down. The calculating results show that the channels form a tilt angle through the interaction between the head group of DPPC with mica substrates. We concluded that the several factors play an important role in the transferring process, such as the interfacial energy, electrostatic interactions and interactions of dipole-dipole between DPPC molecules. Consequently, surface structure consisting of stripes and channels was formed on the mica substrates.

Finally, we consider that withdrawing Langmuir-Blodgett monolayers on mica substrate is a displacement and competing process. Transferring the monolayers onto the mica substrate from the interface between water and monolayers is as important as persuading the adsorbed water to keep away from the monolayers there.

\section{Acknowledgements}

This work was supported by the National Science Foundation of China, Grant No. 21203055 and Start-up funds for high-level introduction of talents for scientific research in Shanghai Publishing and Printing College. 


\section{Conflicts of Interest}

The authors declare no conflicts of interest regarding the publication of this paper.

\section{References}

[1] Gaines, G.L. (1996) Insoluble Monolayers at Liquid-Gas Interface. Wiley-Interscience, New York.

[2] Brockman, H. (1999) Lipid Monolayers: Why Use Half a Membrane to Characterize Protein-Membrane Interactions? Current Opinion in Structural Biology, 9, 438-443. https://doi.org/10.1016/S0959-440X(99)80061-X

[3] Ouyang, J.-M. and Deng, S.-P. (2003) Controlled and Uncontrolled Crystallization of Calcium Oxalate Monohydrate in the Presence of Citric Acid. Dalton Transactions, No. 14, 2846-2851. https://doi.org/10.1039/b304319c

[4] von Tschaner, V. and McConnell, H.M. (1981) An Alternative View of Phospholipid Phase Behavior at the Air-Water Interface Microscope and Film Balance Studies. Biophysical Journal, 36, 409-419. https://doi.org/10.1016/S0006-3495(81)84740-6

[5] von Tschaner, V. and McConnell, H.M. (1981) Physical Properties of Lipid Monolayers on Alkylated Planar Glass Surfaces. Biophysical Journal, 36, 421-427. https://doi.org/10.1016/S0006-3495(81)84741-8

[6] Florsheimer, M. and Mohwald, H. (1990) Growth of Large Liquid Crystalline Domains of Phospholipids at Air-Water Interfaces. Thin Solid Films, 189, 379-387. https://doi.org/10.1016/0040-6090(90)90467-R

[7] Kjaer, K., Als-Nielsen, J., Helm, C.A., Laxhuber, L.A. and Mohwald, H. (1987) Ordering in Lipid Monolayers Studied by Synchrotron X-Ray Diffraction and Fluorescence Microscopy. Physical Review Letters, 58, 2224. https://doi.org/10.1103/PhysRevLett.58.2224

[8] Kjaer, K., Als-Nielsen, J., Helm, C.A., Tippmann-Krayer, P. and Mohwald, H. (1988) An X-Ray Scattering Study of Lipid Monolayers at the Air-Water Interface and on Solid Supports. Thin Solid Films, 159, 17-28.

https://doi.org/10.1016/0040-6090(88)90613-X

[9] Fischer, A. and Sackmann, E. (1985) A Charge-Decoration Technique for Studying the Heterogeneity of Coexistent Monolayer Phases by Electron Microscopy. Nature, 313, 299-301. https://doi.org/10.1038/313299a0

[10] Yang, X.-M., Xiao, D., Lu, Z.-H. and Wei, Y. (1995) Structural Investigation of Langmuir-Blodgett Monolayers of L- $\alpha$-dipalmitoylphosphatidylcholine by Atomic Force Microscopy. Applied Surface Science, 90, 175-183.

https://doi.org/10.1016/0169-4332(95)00067-4

[11] Singh, S. and Keller, D.J. (1991) Atomic Force Microscopy of Supported Planar Membrane Bilayers. Biophysical Journal, 60, 1401-1410. https://doi.org/10.1016/S0006-3495(91)82177-4

[12] Brandow, S.L., Turner, D.C., Ratna, B.R. and Gaber, B.P. (1993) Modification of Supported Lipid Membranes by Atomic Force Microscopy. Biophysical Journal, 64, 898-902. https://doi.org/10.1016/S0006-3495(93)81450-4

[13] Kumar, A., Biebuyk, H.A. and Whitesides, G.M. (1994) Patterning Self-Assembled Monolayers: Applications in Materials Science. Langmuir, 10, 1498-1511. https://doi.org/10.1021/la00017a030 
[14] Xia, Y. and Whitesides, G.M. (1997) Extending Microcontact Printing as a Microlithographic Technique. Langmuir, 13, 2059-2067. https://doi.org/10.1021/la960936e

[15] Ewans, S.D., Flynn, T.M. and Ulman, A. (1995) Self-Assembled Multilayer Formation on Predefined Templates. Langmuir, 11, 3811-3814.

https://doi.org/10.1021/la00010a035

[16] Abott, N.L., Folkers, J.P. and Whitesides, G.M. (1992) Manipulation of the Wettability of Surfaces on the 0.1 - to 1-Micrometer Scale through Micromachining and Molecular Self-Assembly. Science, 257, 1380-1382. https://doi.org/10.1126/science.257.5075.1380

[17] Wang, R., et al. (1997) Light-Induced Amphiphilic Surfaces. Nature, 388, 431-432. https://doi.org/10.1038/41233

[18] Calvert, J.M. (1993) Lithographic Patterning of Self-Assembled Films. Journal of Vacuum Science \& Technology B, 11, 2155. https://doi.org/10.1116/1.586449

[19] Gau, H., Herminghaus, S., Lenz, P. and Lipowsky, R. (1999) Liquid Morphologies on Structured Surfaces: From Microchannels to Microchips. Science, 283, 46-49. https://doi.org/10.1126/science.283.5398.46

[20] Wittmann, J.C. and Smith, P. (1991) Highly Oriented Thin Films of Poly(tetrafluoroethylene) as a Substrate for Oriented Growth of Materials. Nature, 352, 414-417. https://doi.org/10.1038/352414a0

[21] Hans, R. and Karl, S. (1992) Structural Changes in Lipid Monolayers during the Langmuir-Blodgett Transfer due to Substrate/Monolayer Interactions. Thin Solid Films, 210-211, 9. https://doi.org/10.1016/0040-6090(92)90153-3

[22] Yuan, C., et al. (1996) Lanthanide Ion Induced Formation of Stripes Domain Structure in Phospholipid Langmuir-Blodgett Monolayer Film Observed by Atomic Force Microscopy. Surface Science, 366, L729-L734.

https://doi.org/10.1016/0039-6028(96)00887-4

[23] Gleche, M., Chi, L.F. and Fuchs, H. (2000) Nanoscopic Channel Lattices with Controlled Anisotropic Wetting. Nature, 403, 173-175. https://doi.org/10.1038/35003149

[24] Peters, R., Brünger, A. and Schulten, K. (1981) Continuous Fluorescence Microphotolysis: A Sensitive Method for Study of Diffusion Processes in Single Cells. Proceedings of the National Academy of Sciences of the United States of America, 78, 962-966. https://doi.org/10.1073/pnas.78.2.962

[25] McConnell, H.M., Tamm, L.K. and Weis, R.M. (1984) Periodic Structures in Lipid Monolayer Phase Transitions. Proceedings of the National Academy of Sciences of the United States of America, 81, 3249-3253. https://doi.org/10.1073/pnas.81.10.3249

[26] Petrov, J.G., Kuhn, H. and Möbius, D. (1980) Three-Phase Contact Line Motion in the Deposition of Spread Monolayers. Journal of Colloid and Interface Science, 73, 66-75. https://doi.org/10.1016/0021-9797(80)90123-X

[27] Spratte, K., Chi, L.F. and Riegler, H. (1994) Physisorption Instabilities during Dynamic Langmuir Wetting. Europhysics Letters, 25, 211-217. https://doi.org/10.1209/0295-5075/25/3/010

[28] Weis, R.M. and McConnell, H.M. (1985) Cholesterol Stabilizes the Crystal-Liquid Interface in Phospholipid Monolayers. Journal of Physical Chemistry, 89, 4453-4459. https://doi.org/10.1021/j100267a011 